\title{
Schwannoma of The Nasal Septum with Degenerative Changes: A Case Report
}

\author{
N Sadiya \\ Department of Histopathology, Apollo Speciality Hospital, Vanagaram, Chennai, Tamil Nadu, India
}

\section{ABSTRACT}

Tumors of the sinonasal tract are usually of epithelial in origin such as papilloma, salivary gland tumors or carcinoma. Soft tissue tumors of the sinonasal tract are rare and are predominantly vascular and fibrohistiocytic in origin. Neural tumors are unusual in this location.Schwannomas of the sinonasal tract are rare, benign, slow growing peripheral nerve sheath tumors representing less than $4 \%$ of the schwannomas of the head and neck region without age, race or gender predilection. We present a case of a 50 year old female patient with history of unilateral nasal obstruction and with a polypoidal mass of the right nasal septum, in whom a diagnosis of Schwannoma was rendered.

\section{Keywords: Schwannoma, Nasal Septum, Degenerative Changes}

\section{Introduction}

Schwannomas are slow growing benign tumors and originate from Schwann cells in the sheath of the myelinated nerve fibres. Stout (1935) coined the term Neurilemmoma because of its origin from cells of nerve sheath. They are relatively common in the head and neck $(25-45 \%)$ region but involvement of the sinonasal region is rare constituting ${ }_{[2,]^{4} \%}{ }^{[1]}$. Localization to nasal septum is exceedingly rare . Usually schwannomas arise from large peripheral nervefibers such as vestibulocochlear nerve, vagal nerve and cervical sympathetic trunk ${ }^{[3]}$. However in our case due to the localization of the tumor; it may be considered to derive from small somatosensorial nerve fibers of nasal septum (e.g. nasopalatine or nasociliary nerves). The signs and symptoms associated with sinonasal schwannomas are rhinorrhea, epistaxis, anosmia and facial swelling. As these tumors are located in a cavity they are able to grow silently to a substantial size before diagnosis. Surgical resection is the treatment of choice.

\section{Case Report}

A 50 year old lady was referred to our hospital with a six month history of progressive right sided nasal obstruction. There was no history of anosmia, epistaxis or head ache. Patient was neither suffering from any comorbid disease. Anterior rhinoscopy revealed a polypoidal mass filling the right nasal cavity with no bleeding on touch. The nasal septum was deviated to the opposite side and left nasal cavity was clear. Computerized tomography $[\mathrm{CT}]$ of the paranasal sinuses revealed a polypoidal mass measuring $45 \times 13 \times 10 \mathrm{~mm}$ arising from the right side of the nasal septum. The lesion was well circumscribed with smooth margins with no evidence of bone destruction. The paranasal sinuses were unremarkable. The mass was completely excised. Gross examination revealed multiple polypoidal soft tissue bits totally aggregating to $5 \times 1.5 \times 1 \mathrm{cms}$.

Microscopic examination revealed nasal mucosa lined by ciliated columnar type of epithelium. The sub epithelium showed edema and a fairly circumscribed spindle cell neoplasm [Fig 1] with hyper and hypocellular areas arranged as fascicles, [Fig $2 \& 3$ ] sheets and in vague nodular aggregates. The neoplasm was composed of oval to spindle shaped nuclei with coarse chromatin at foci showing distinct nucleoli with moderate eosinophilic cytoplasm and few showing intranuclear inclusion. Scattered mitosis rating to less than $4 / 10 \mathrm{hpf}$ was noted. At foci bizarre nuclear atypia with hyperchromasia was seen [Fig 3 inset]. The surrounding stroma showed myxoid change with lymphoplasmacytic infiltrate and hyalinized blood vessels. No evidence of necrosis identified.

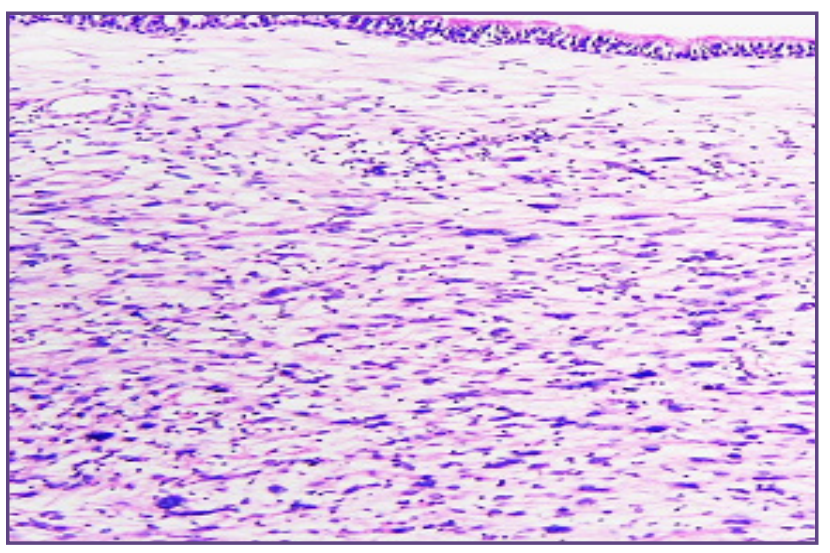

Fig. 1: Microphotograph showing nasal mucosa lined by ciliated columnar epithelium with a sub epithelial spindle cell neoplasm (H \& E -10X). 
Immunohistochemistry was done, which showed diffuse positivity for S100 with negativity for Desmin, Vimentin, SMA and EMA. Ki -67 proliferative index was 3.5\% [Fig 4].

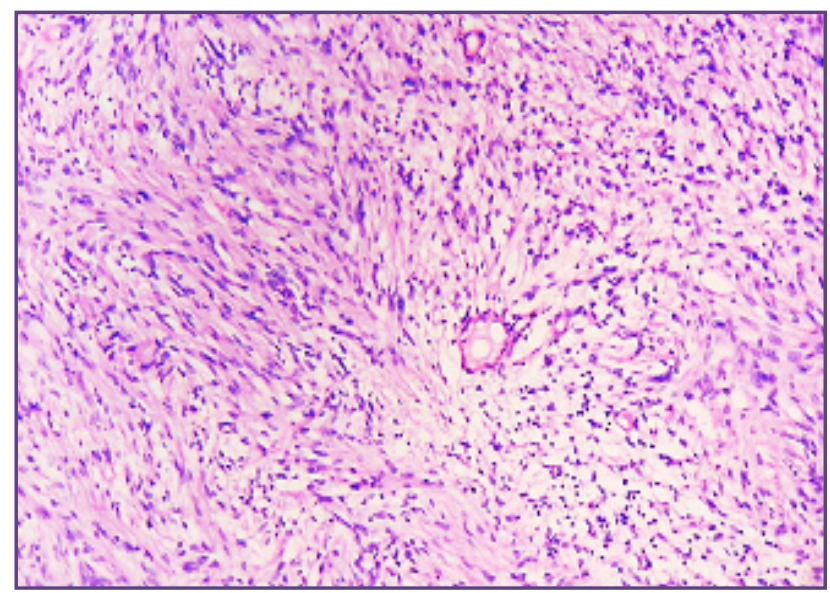

Fig. 2: Microphotograph showing hyper and hypocellular areas with congested blood vessels (H\&E -20X).
A diagnosis of Schwannoma with degenerative changes was rendered. Patient was under regular follow up for one year and there was no recurrence after one year follow up.

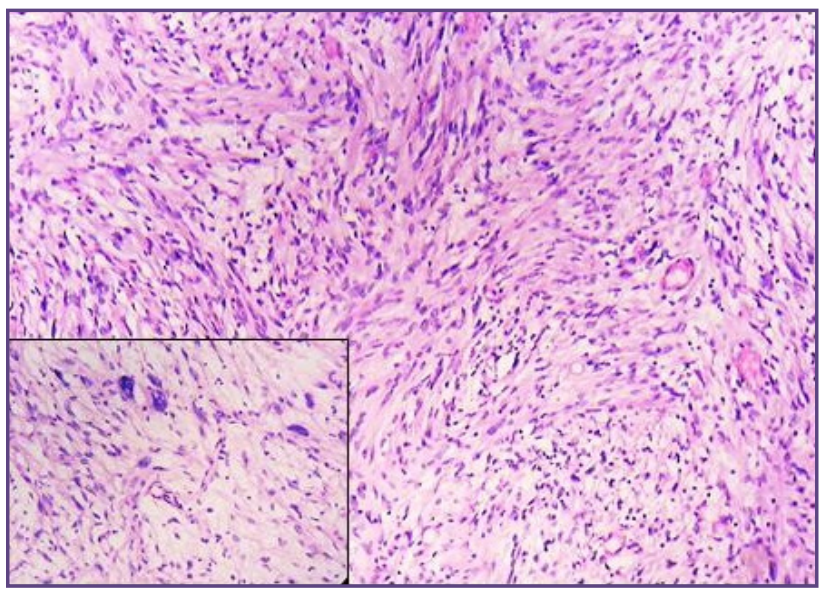

Fig. 3: Photomicrograph showing spindle cells arranged in fascicles with inset showing degenerative nuclear atypia. (H\&E - 40X).
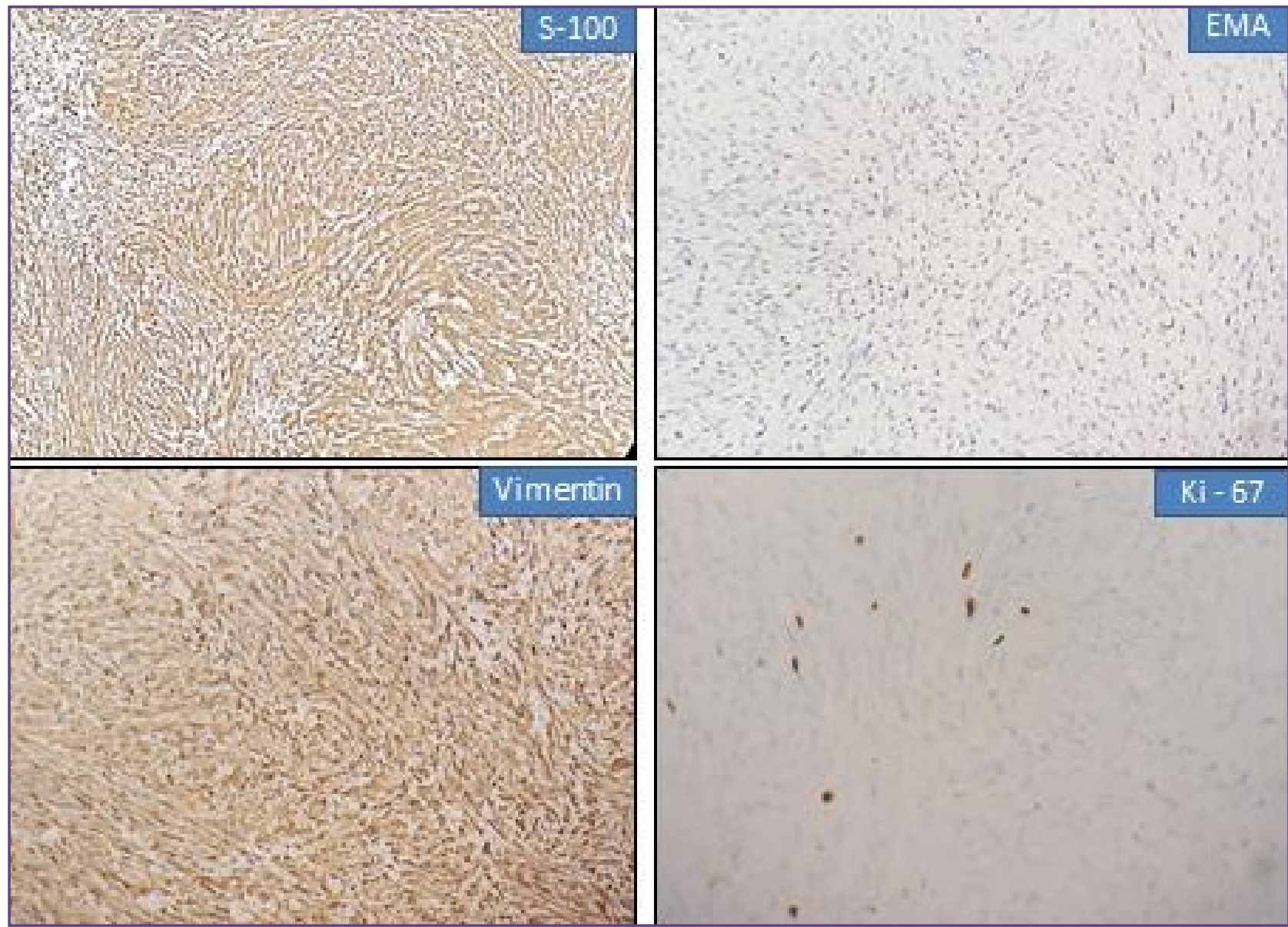

Fig. 4: Photomicrograph in Immunohistochemistry showing diffuse positivity for S100 and Vimentin with negativity for EMA(IHC 10X).Ki-67 proliferative index was 3.5\%(IHC 40X). 


\section{Discussion}

Sinonasal schwannomas are uncommon tumors that have been described as case reports or as small case series. To date, only 20 reports of nasal septum schwannoma have been reported in western literature ${ }^{[4]}$. Schwannomas of the nasal septum are extremely unusual and not many cases have been reported previously. The literature mentions approximately only 70 cases of nose and paranasal sinus schwannomas that are mostly seen in adults aged 40-60 years and without gender or racial predilection ${ }^{[5]}$. Nasal Schwannomas are presumed to be arising from the sheath of the ophthalmic and maxillary branches of the trigeminal nerve and autonomic ganglia [6]. Sinonasal schwannomas have no age, race or gender predilection. There is no apparent site predilection on the septum. Nasal obstruction is the most common clinical symptom followed by epistaxis [7]. CT scan delineates the soft tissue nature of the lesion, outlines the skeletal margins and offers better resolution of bone invasion than Magnetic resonance imaging [MRI].

The main differential diagnosis of nasal masses other than Schwannomas includes Nasal polyps, Neurofibroma, Malignant peripheral nerve sheath tumor, Fibromyxoma, Leiomyoma and Lobular capillary haemangioma. Histopathological examination with ancillary studies like Immunohistochemistry offers a definitive diagnosis. The histopathological features of a spindle cell neoplasm with diffuse nuclear and cytoplasmic positivity for S100 confirmed the diagnosis of Schwannoma. The negativity for Desmin, SMA, EMA and Vimentin ruled out the diagnosis of leiomyoma and other spindle cell neoplasms. The absence of circumscribed nodular proliferation of capillary sized blood vessels ruled out Lobular capillary haemangioma. Neurofibromas also originate from Schwann cells, are sub mucosal and must be considered as differential. However, neurofibromas show spindle cells with wavy or buckled nuclei, indistinct cytoplasm and absence of Verocay bodies. An associated collagenized and or myxoid stromal component is present. Neoplastic cells are S100 protein positive but the intensity of staining is less than that seen in Schwannomas. Meningiomas may be encountered in sinonasal location. It is characterized by whorls of tumor cells, psammoma bodies and stains with EMA. The presence of hypercellularity and atypia may lead to the diagnosis of these lesions as sarcomas. Dahl reported 6 out of 11 cases which have been misdiagnosed as sarcoma $[8]$.

The first case of Ancient Schwannoma of the head and neck region was reported by Eversoli and Howell in $1971^{[9]}$. Since the first report several authors have described Ancient Schwannomas in a variety of locations in the head and neck region, involving the oral cavity, sub mandibular gland, pharynx orbit and infratemporal fossa. Those Schwannomas designated as ancient show cystic or fatty degeneration with hyperchromasia and these histopathological features are attributed to the long duration of Schwannoma ${ }^{[10]}$

The incidence of Malignant Schwannoma is 2\%. Malignant changes in a benign schwannoma are extremely rare. The diagnosis of a malignant change in a benign schwannoma is based on the following criteria 1) Demonstrable areas of benign schwannoma .2) Unequivocal malignant foci manifested by increased cellularity, numerous mitosis ( $>5 / 10 \mathrm{hpf}$ ), anaplastic cells and invasiveness. 3 ) Transitional areas between malignant and benign regions.4) Absence of clinical evidence of Neurofibromatosis I.

\section{Conclusion}

Sinonasal region is an uncommon location fora Schwannoma to present, but their existence should be realized and should be considered in the differential diagnosis of a benign appearing nasal cavity mass. Histopathological examination with immunohistochemistry is required for a definitive diagnosis.

\section{References}

1. Sheikh HY, Chakravarthy RP, Selvin NJ, Sykes AJ, Banerjee SS. Benign Schwannoma in paranasal sinuses: a clinicopathological study of five cases, emphasizing diagnostic difficulties. J Laryngol Otol 2008; 122 (6) : 598602

2. Luchi GER, Magalha es MR, Lanzelotti SM, et al. Nasal schwannoma. Braz J Otorhinolaryngol.. 2006;72(5):714.

3. Cadd B, Offiah C, Alusi G. A surprising cause of unilateral nasal obstruction and epistaxis: nasal septal schwannoma. J Surg Case Rep.. 2014; 21:2014.

4. Pagilla F, Giourgo G, Matte E, Colombo A. An asymptomatic Schwannoma of nasal septum. Report of a unique case. Ear Nose Throat.J.2009; 88:1264-1265.

5. Pauna HF, Carvalho GM, Guimaraes AC, Maunsell RC, Sakano E. Schwannoma of the nasal septum: evaluation of unilateral nasal mass. Braz J Otorhinolaryngol 2013; 79: 403.

6. Batsakis J. Tumors of the Head and Neck. Clinical and Pathological Considerations.2 nd ed. Baltimore MD. Williams \& Wilkins; 1979: 313 -333.

7. OiH, Watanabe $Y$, Shojaku H, et al. Nasal septal neurinoma .Acta Otolaryngol (Stockh). 1993 (suppl. 504);151-154.

8. Jackson RM, Rice DH, Crockett DM. Malignant schwannoma of the cervical vagus nerve in a patient 
with neurofibromatosis (von Recklinghausen's disease). Otolaryngol Head Neck Surg. 1986; 94:641-644.

9. Eversole LR, Howell RM. Ancient neurilemmoma of the oral cavity. Oral Surg.1971; 32:440 -443.
10. Bhat SP, Permi HS, Shetty R, Shenoy J, Prasad K, Hegde P.Ancient schwannoma of the chest wall diagnosed by fine needleaspiration cytology - a rare case report. Nitte Univ J Heal Sci.2012;2:27- 29 .

*Corresponding author:

Dr. N.Sadiya, Door No 21, Flat No T1, Ameer Regency, Balaji Nagar, 2ND Street, Royapettah, Chennai -14 Pin - 600014 Tamil Nadu, India

Email: shajanabasraar@gmail.com

Financial or other Competing Interests: None. 